\title{
Simple functional tests in COPD: stand up and be counted!
}

\author{
Matthew Maddocks ${ }^{1}$, Claire M. Nolan ${ }^{2,3}$ and William D-C. Man ${ }^{2,3}$
}

Affiliations: ${ }^{1}$ King's College London, Cicely Saunders Institute, London, UK. ${ }^{2}$ NIHR Respiratory Biomedical Research Unit, Royal Brompton and Harefield NHS Foundation Trust and Imperial College London, London, UK. ${ }^{3}$ Harefield Pulmonary Rehabilitation Unit, Harefield Hospital, Harefield, UK.

Correspondence: Dr William Man, Harefield Pulmonary Rehabilitation Unit, Dept of Respiratory Medicine, Harefield Hospital, Hill End Road, Harefield, UB9 6JH, UK. E-mail: w.manđarbht.nhs.uk

@ERSpublications

Simple functional tests in COPD: sit to stand and gait speed can rapidly assess physical performance in COPD http://ow.ly/jIRJ308ZsBg

Cite this article as: Maddocks M, Nolan CM, Man WD-C. Simple functional tests in COPD: stand up and be counted!. Eur Respir J 2017; 49: 1700104 [https://doi.org/10.1183/13993003.00104-2017].

Chronic obstructive pulmonary disease (COPD) is widely recognised as a multisystem disorder [1]. Although forced expiratory volume in $1 \mathrm{~s}(\mathrm{FEV} 1)$ is a widely accepted marker used in the diagnosis and staging of COPD, it tells us very little about extra-pulmonary manifestations, such as skeletal muscle weakness and sarcopenia [2-4], and shares only a weak relationship with patient-centred outcomes of health-related quality of life and exercise capacity [5]. Pulmonary rehabilitation, the most effective non-pharmacological intervention in COPD, brings meaningful benefits to the patient, yet has little effect on FEV1. Hence, additional assessment tools are needed to provide a comprehensive picture of disease impact.

Exercise intolerance and functional impairment are commonly reported by patients with COPD. They result from multiple contributing factors including dynamic hyperinflation, increased respiratory load, poor gas exchange, skeletal muscle dysfunction, age-related frailty, physical de-conditioning, mood disturbance, lack of motivation and comorbidity burden. Physical functioning may therefore reflect the impact of COPD better than FEV1. Certainly, reduced exercise capacity has been associated consistently with increased mortality $[6,7]$.

So how best to routinely and objectively assess physical functioning? Laboratory-based cardiopulmonary exercise testing (CPET), typically using a cycle ergometer or treadmill, is the physiological gold standard, but is time-consuming and requires specialist equipment and expertise, making it impractical for routine use in a busy clinic setting. Field walking tests, such as the 6-min walk test (6MWT), incremental walk test and endurance shuttle walk test, are simpler and cheaper to perform than CPET, and have been readily adopted by the pulmonary rehabilitation community. Increasing evidence support their validity and responsiveness $[8,9]$, and technical standards have recently been published [10]. However, even these tests may not always be possible to complete in some settings. For example, the 6MWT requires a course of at least $30 \mathrm{~m}$, which is rarely feasible in the patient's home, acute hospital bedside or the outpatient clinic.

Recently, there has been growing interest in even simpler functional tests, particularly lower limb physical performance measures, to assess people with chronic respiratory disease [11]. Many of these tests have their origins in gerontology, for use in large community-based epidemiological studies of older adults, where rapid and practical assessment of physical functioning is required in the home setting. Broadly, there are

Received: Jan 162017 | Accepted: Jan 242017

Support statement: M. Maddocks is supported by Cicely Saunders International and the National Institute for Health Research (NIHR) Collaboration for Leadership in Applied Health Research and Care (CLAHRC) South London. C.M. Nolan is supported by a NIHR Doctoral Research Fellowship (DRF-2014-07-089). W. Man is supported by the NIHR CLAHRC for Northwest London. The views expressed in this publication are those of the authors and not necessarily those of the NHS, the NIHR nor the Department of Health.

Conflict of interest: Disclosures can be found alongside this article at erj.ersjournals.com

Copyright @ERS 2017 
four main groups of simple lower limb physical performance measures: 1) gait speed tests (time to walk a set distance) $[12,13]$; 2) sit-to-stand (STS) tests, which measure either time taken to stand from a sitting position five times (known as the five-repetition STS) [14] or the number of sit to stands in a set time (typically $1-3 \mathrm{~min}$ ) $[15,16]$; 3) step tests (many protocols exist but the best described is the 6-min step test, for which the person is asked to step up and down as many times as possible in 6 min) [17]; and 4) the timed up-and-go test [18], which incorporates elements of both gait speed and STS tests.

In this issue of the European Respiratory Journal, Своок et al. [19] provide a psychometric evaluation of the 1-min STS test. The authors should be congratulated for a comprehensive evaluation, which incorporated assessment of learning effect, test-retest reliability, construct validity, responsiveness and minimal important difference (MID) of the 1-min STS test, using data from two longitudinal studies involving 52 and 203 patients with COPD undergoing pulmonary rehabilitation [19]. The 1-min STS test had minimal learning effect (ICC 0.93) with excellent test-retest reliability (interclass correlation coefficient 0.99). Both before and after rehabilitation, there was a strong relationship between the 1-min STS and the $6 \mathrm{MWT}$, and the 1-min STS test was responsive to rehabilitation. Using anchor and distribution methods, the MID was estimated to be three STS manoeuvres when prioritising the anchor-based method. This study corroborates previous data supporting the validity and responsiveness of sit-to-stand tests [14]. JonEs et al. [14] showed similar excellent reliability, validity and responsiveness to pulmonary rehabilitation of the five-repetition STS, with the authors conservatively estimating the MID to be $1.7 \mathrm{~s}$.

STS tests have obvious advantages. They require little space, simple equipment (chair and a stopwatch), and are feasible in all settings, including the home, busy clinic setting or the acute hospital bedside. Is there a need for more formal field walking exercise tests or laboratory-based CPET? In a small sub-group, the authors performed the 1-min STS and 6MWT whilst metabolic data were collected to examine physiological responses. End-exercise oxygen uptake, ventilation parameters, respiratory rate and heart rate were similar. This might suggest the 1-min STS is a test of exercise capacity, but really only confirms the concurrent validity of STS and field walking tests under the umbrella of physical functioning tests. Indeed, fewer patients desaturated during the 1-min STS, and the correlation between change values in the tests from before to after rehabilitation was low $(n=52: r=-0.08, n=203: r=0.21)$ indicating that the 1-min STS and 6MWT measure different constructs. Similarly, Jones et al. [14] showed only weak relationships between changes in five-repetition STS and incremental shuttle walk with pulmonary rehabilitation. Longer variants of the STS may be a better reflection of exercise capacity [16]. AguiLANIU et al. [16] demonstrated that the physiological responses and symptom burden of a semi-paced 3-min chair rise test were interchangeable with the 6MWT in most patients.

A limitation of the study (acknowledged by the authors), and of STS tests in general, is the presence of a floor effect. In the study by СRоок et al. [19] patients unable to perform five STS manoeuvres were excluded. The number of patients excluded for this reason was not reported, but in a previous study of outpatients attending pulmonary rehabilitation, $15 \%$ were unable to meet this threshold [14]. Indeed between $9 \%$ and $14 \%$ of outpatients are not able to even stand unaided once [20]. Therefore, only selected, higher-functioning patients are able to perform STS tests, excluding those with the poorest functional ability or lower limb impairment. This somewhat limits the advantage of this test as a feasible functional outcome measure in the home or acute hospital bedside, settings where one might expect to find patients with the poorest physical functioning and greatest skeletal muscle dysfunction. However, for these patients, it is important to note that floor effects may exist in other tests of exercise capacity, such as cardiopulmonary exercise testing and field walking tests. In this group, gait speed tests (typically over a short distance such as $4 \mathrm{~m}$ ) may provide a better fit to the population of interest $[3,12,13,21,22]$.

Accepting that STS tests may not measure exercise capacity, and have utility as an outcome measure in only selected patients with COPD, simple functional tests remain helpful to the clinician. As "global" markers of disease impact, they can be used to stratify patients rapidly. For example, outpatients with COPD and "slow" 4-m gait speed $\left(<0.8 \mathrm{~m} \cdot \mathrm{s}^{-1}\right)$ have significantly reduced exercise capacity and report higher respiratory disability and worse health-related quality of life compared with those with preserved walking speed, despite similar $\mathrm{FEV}_{1} \%$ predicted [13]. Gait speed is also widely incorporated into consensus definitions of geriatric syndromes such as sarcopenia and frailty [3, 22, 23]. Moreover, in line with the gerontology literature, there is increasing evidence to support the association between functional performance and risk of adverse outcomes in COPD. The 1-min STS has previously been shown to be strongly associated with mortality in COPD, with an adjusted hazard ratio for 2-year mortality of 0.58 (95\% CI 0.40-0.85) for every five additional repetitions [24], while Kon et al. [25] recently demonstrated that the 4-m gait speed was not only feasible in patients hospitalised with acute exacerbation of COPD, but also independently predicted hospital readmission for older patients. Simple functional tests have been incorporated into the baseline evaluation of several COPD cohort studies [22, 26, 27], and longitudinal prognostic data are anticipated. 
Simple functional tests are here to stay, and will become increasingly relevant in COPD and other chronic respiratory diseases, particularly with an ageing population, increasing multi-morbidity and frailty, and more people living with prolonged impairment and disability [22, 23]. Well-conducted psychometric studies, such as that described by CROOK et al. [19], are important in helping clinicians understand the relevance of test values, and give them greater confidence to incorporate these tests more widely into the routine clinical assessment of their patients.

\section{References}

1 Barnes PJ, Celli BR. Systemic manifestations and comorbidities of COPD. Eur Respir J 2009; 33: 1165-1185.

2 Donaldson AV, Maddocks M, Martolini D, et al. Muscle function in COPD: a complex interplay. Int J Chron Obstruct Pulmon Dis 2012; 7: 523-535.

3 Jones SE, Maddocks M, Kon SS, et al. Sarcopenia in COPD: prevalence, clinical correlates and response to pulmonary rehabilitation. Thorax 2015; 70: 213-218.

4 Bone AE, Hepgul N, Kon S, et al. Sarcopenia and frailty in chronic respiratory disease: Lessons from gerontology. Chron Respir Dis 2017; 14: 85-99.

5 Jones PW, Agusti AG. Outcomes and markers in the assessment of chronic obstructive pulmonary disease. Eur Respir J 2006; 27: 822-832.

6 Polkey MI, Spruit MA, Edwards LD, et al. Six-minute-walk test in chronic obstructive pulmonary disease: minimal clinically important difference for death or hospitalization. Am J Respir Crit Care Med 2013; 187: 382-386.

7 Ringbaek T, Martinez G, Brondum E, et al. Shuttle walking test as predictor of survival in chronic obstructive pulmonary disease patients enrolled in a rehabilitation program. J Cardiopulm Rehabil Prev 2010; 30: 409-414.

8 Singh SJ, Puhan MA, Andrianopoulos V, et al. An official systematic review of the European Respiratory Society/ American Thoracic Society: measurement properties of field walking tests in chronic respiratory disease. Eur Respir J 2014; 44: 1447-1478.

9 Puente-Maestu L, Palange P, Casaburi R, et al. Use of exercise testing in the evaluation of interventional efficacy: an official ERS statement. Eur Respir J 2016; 47: 429-460.

10 Holland AE, Spruit MA, Troosters T, et al. An official European Respiratory Society/American Thoracic Society technical standard: field walking tests in chronic respiratory disease. Eur Respir J 2014; 44: 1428-1446.

11 Bisca GW, Morita AA, Hernandes NA, et al. Simple lower limb functional tests in patients with chronic obstructive pulmonary disease: a systematic review. Arch Phys Med Rehabil 2015; 96: 2221-2230.

12 Kon SS, Canavan JL, Nolan CM, et al. The 4-metre gait speed in COPD: responsiveness and minimal clinically important difference. Eur Respir J 2014; 43: 1298-1305.

13 Kon SS, Patel MS, Canavan JL, et al. Reliability and validity of 4-metre gait speed in COPD. Eur Respir J 2013; 42: $333-340$.

14 Jones SE, Kon SS, Canavan JL, et al. The five-repetition sit-to-stand test as a functional outcome measure in COPD. Thorax 2013; 68: 1015-1020.

15 Strassmann A, Steurer-Stey C, Lana KD, et al. Population-based reference values for the 1-min sit-to-stand test. Int J Public Health 2013; 58: 949-953.

16 Aguilaniu B, Roth H, Gonzalez-Bermejo J, et al. A simple semipaced 3-minute chair rise test for routine exercise tolerance testing in COPD. Int J Chron Obstruct Pulmon Dis 2014; 9: 1009-1019.

17 Grosbois JM, Riquier C, Chehere B, et al. Six-minute stepper test: a valid clinical exercise tolerance test for COPD patients. Int J Chron Obstruct Pulmon Dis 2016; 11: 657-663.

18 Mesquita R, Wilke S, Smid DE, et al. Measurement properties of the timed up and go test in patients with COPD. Chron Respir Dis 2016; in press [https://doi.org/10.1177/1479972316647178].

19 Crook S, Büsching G, Schultz K, et al. A multicentre validation of the 1-min sit-to-stand test in patients with COPD. Eur Respir J 2017; 49: 1601871.

20 Canavan JL, Maddocks M, Nolan CM, et al. Functionally relevant cut point for isometric quadriceps muscle strength in chronic respiratory disease. Am J Respir Crit Care Med 2015; 192: 395-397.

21 Kon SS, Canavan JL, Nolan CM, et al. What the 4-metre gait speed measures and why it cannot replace functional capacity tests. Eur Respir J 2014; 43: 1820-1822.

22 Maddocks M, Kon SS, Canavan JL, et al. Physical frailty and pulmonary rehabilitation in COPD: a prospective cohort study. Thorax 2016; 71: 988-995.

23 Bousquet J, Dinh-Xuan AT, Similowski T, et al. Should we use gait speed in COPD, FEV1 in frailty and dyspnoea in both? Eur Respir J 2016; 48: 315-319.

24 Puhan MA, Siebeling L, Zoller M, et al. Simple functional performance tests and mortality in COPD. Eur Respir J 2013; 42: 956-963.

25 Kon SS, Jones SE, Schofield SJ, et al. Gait speed and readmission following hospitalisation for acute exacerbations of COPD: a prospective study. Thorax 2015; 70: 1131-1137.

26 Mohan D, Gale NS, McEniery CM, et al. Evaluating the role of inflammation in chronic airways disease: the ERICA study. COPD 2014; 11: 552-559.

27 Gale NS, Albarrati AM, Munnery MM, et al. Assessment of Risk in Chronic Airways Disease Evaluation (ARCADE): protocol and preliminary data. Chron Respir Dis 2014; 11: 199-207. 